\title{
Novel ACLV1 Mutation Identified in Late Onset Hereditary Hemorrhagic Telangiectasia
}

\author{
Cory Patrick ${ }^{1}$, Kaitlin McIntyre ${ }^{1}$, Jeremy Ramidial'2, Sano Joa ${ }^{3}$, \\ Vijaykumar Dinsukhlal Zaveri ${ }^{3}$, Damien Hansra ${ }^{3,4}$ \\ ${ }^{1}$ Miller School of Medicine, University of Miami, Miami, FL, USA \\ 2Jackson Memorial Hospital, Miami, FL, USA \\ ${ }^{3}$ Mercy Hospital, Miami, FL, USA \\ ${ }^{4}$ Oncology and Radiation Associates, Miami, FL, USA \\ Email: dmhansra@gmail.com
}

Received 22 June 2016; accepted 15 July 2016; published 18 July 2016

Copyright (C) 2016 by authors and Scientific Research Publishing Inc.

This work is licensed under the Creative Commons Attribution International License (CC BY).

http://creativecommons.org/licenses/by/4.0/

(c) (;) Open Access

\begin{abstract}
Hereditary Hemorrhagic Telangiectasia (HHT) is an autosomal dominant disorder with variable expressivity. We present a 62-year-old patient with a rare, late-onset disease course featuring a novel mutation in ACVRL1, a signal transducer in the TGF $\beta$ /BMP pathway.
\end{abstract}

\section{Keywords}

Hereditary Hemorrhagic Telaniectasia, Osler-Weber-Rendu Syndrome, Anemia, Mutation

\section{Introduction}

Hereditary Hemorrhagic Telangiectasia (HHT) is an autosomal dominant disorder with variable expressivity frequently presenting with recurrent epistaxis at adolescence. Here, we present a patient (pt) with a rare, lateonset disease course featuring a novel mutation in ACVRL1, a signal transducer in the TGF $\beta /$ BMP pathway.

\section{Case}

62-year-old female who presented 08/26/15 with worsening episodic epistaxis, fatigue, dyspnea on exertion for 10 years. Physical exam revealed upper and lower distal extremity telangiectasias (Figures 1-3) along with numerous tongue lesions (Figure 4). Labs: HGB 9.4 g/dL (low), HCT 30.1\% (low), MCV 78.7 fL (low), RDW 23\% 


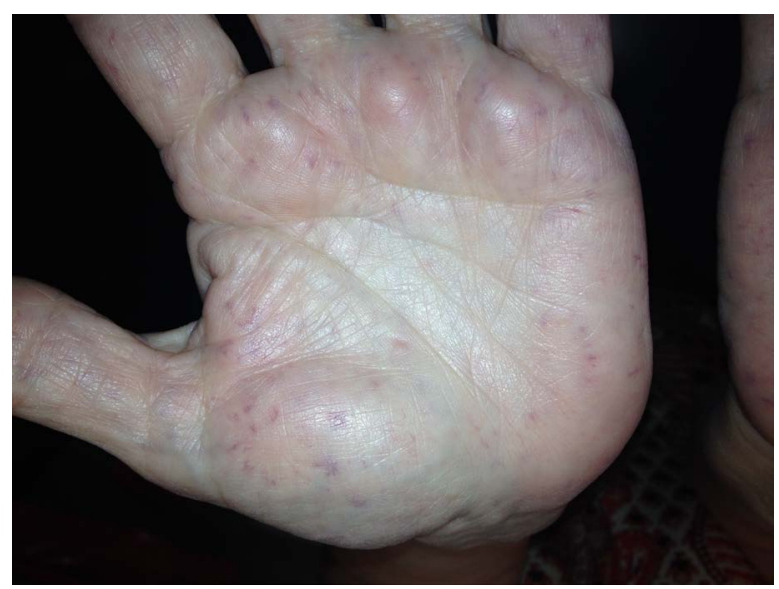

Figure 1. Telangiectasia of right palm.

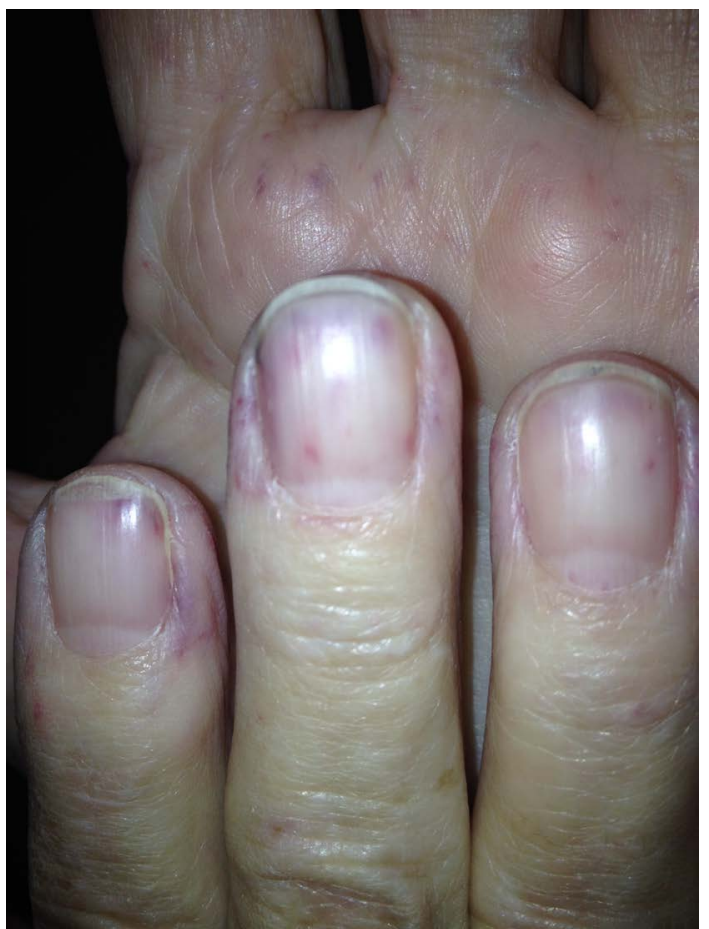

Figure 2. Telangiectasias left finger nails.

(high), Reticulocytes 112700 cells/uL (high), Ferritin 17 ng/mL (low). CMP, PT, PTT, \& fibrinogen were normal. Esophagoduodenoscopy (EGD) revealed non-bleeding gastric antrum arteriovenous malformations (Figure 5). Colonoscopy was normal. Fiberoptic examination by otolaryngology revealed multiple telangiectasias in the nasal mucosa. CT chest abdomen and pelvis 10/1/14 lacked well-defined AV malformations. Targeted sequencing of the exons and exon-intron junctions of known HHT genes, ENG, ACVRL1, SMAD4, RASA1 and BMP9, returned a previously unreported missense mutation in ACVRL1, resulting in a c.998G > A nucleotide substitution and p. Ser333Asn amino acid alteration. The patient was a heterozygote for this alteration. Her final diagnosis is microcytic anemia due to chronic blood loss due to HHT related epistaxis. She was treated with oral iron and periodic ENT cauterizations with stabilization of symptoms and HGB (11.9 g/dL, 9/2/15).

\section{Discussion}

Hereditary Hemorrhagic Telangiectasia (HHT), or Osler-Weber-Rendu syndrome, was first described in the $19^{\text {th }}$ 


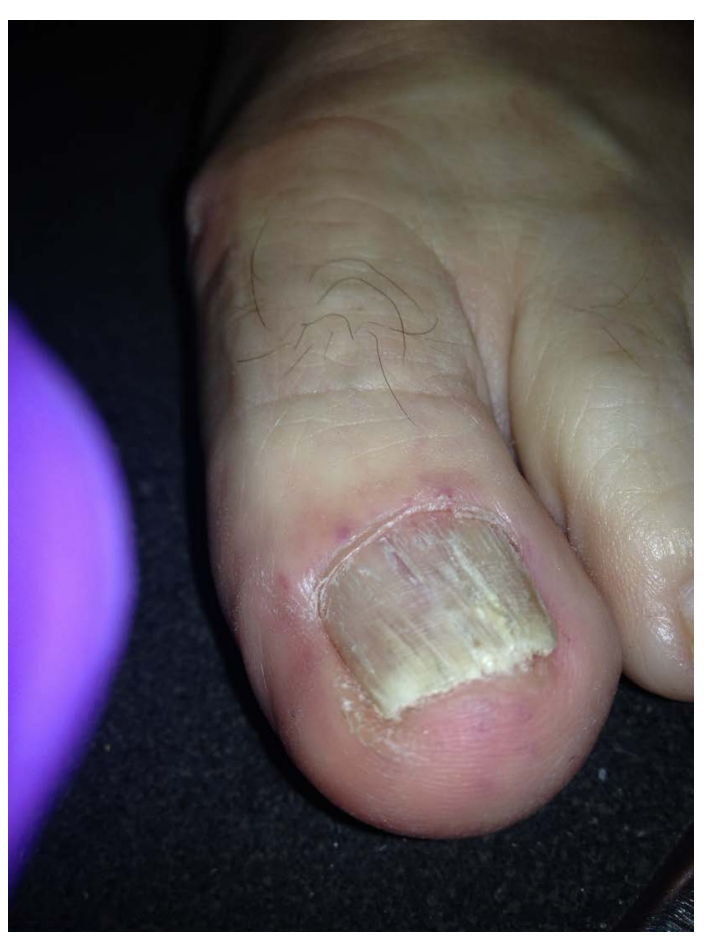

Figure 3. Telangiectasias of left great hallux.

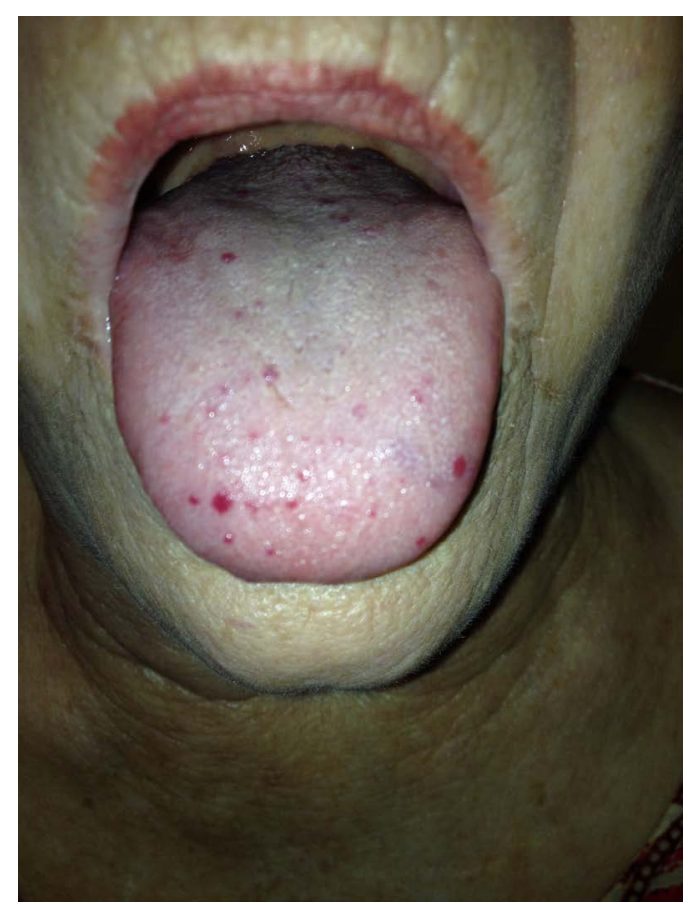

Figure 4. Telangiectasias of the tongue.

century as a hereditary disorder with abnormal vascular structures that caused recurrent bleeding from mucosa throughout the body [1]. Rendu first differentiated this disease from hemophilia when he studied a 52-year-old man with a clinical and family history of anemia, recurrent epistaxis, and telangiectasias. Osler and Weber produced more case reports on similar patients later on that made hereditary hemorrhagic telangiectasia well known within the medical community [2]. 


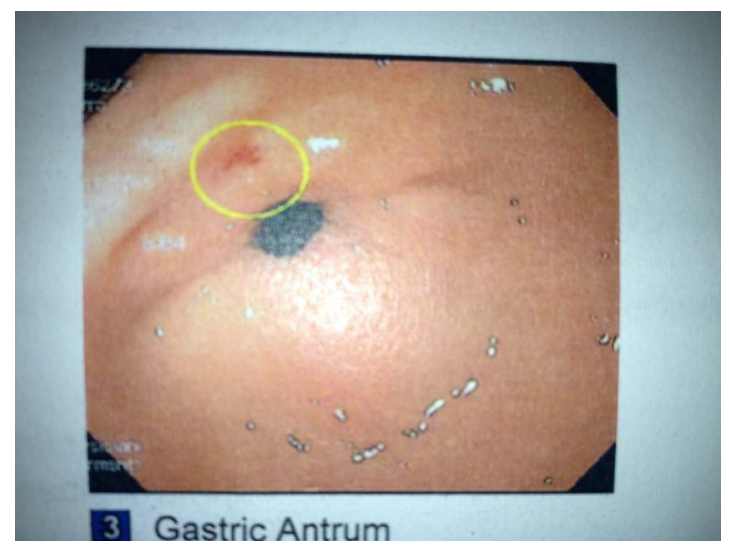

Figure 5. EGD showing gastric antrum AVM.

HHT affects various organs including the nose, skin, lung, brain, and gastrointestinal track. The most common and earliest clinical manifestation is epistaxis from telangiectasias in the nasal mucosa [3]. Over 90 percent of patients with HHT experience their first episode of epistaxis by the age of 21 [4]. Symptoms in the skin, lung, brain, and gastrointestinal track typically present later in the disease course. Common areas for telangiectasias to occur on the skin include the lips, tongue palate, fingers, face, or trunk [3]. Pulmonary arteriovenous malformations are the typical presentation of hereditary hemorrhagic telangiectasia in the respiratory tract. It is estimated that pulmonary arteriovenous malformations are seen in 5 to 15 percent of patients with hereditary hemorrhagic telangiectasia [5]. Neurological symptoms vary and include migraines, transient ischemic attacks, seizures, and hemorrhage [3]. Telangiectasias and hemorrhage are rare in the gastrointestinal track and typically are not symptomatic until the fifth or sixth decade if present. Liver involvement can also occur but is rarely seen [3]. Our patient presents with a late-onset variation of HHT. Epistaxis and other clinical manifestations did not appear until the sixth decade.

The incidence of HHT is approximated to be between 1:5000 and 1:8000. However, the disease is thought to be underreported due to the fact that most patients are unaware of their diagnosis [1].

HHT is an autosomal dominant disorder displaying variable expressivity, locus heterogeneity, and allelic heterogeneity [6]. HHT is attributed to reduction or loss of function alleles in five genes, ENG, ACVRL1, MADH4, BMP9 and BMPR2, resulting in haploinsufficiency of the coded protein [1] [6]. These five genes are all involved in TGF- $\beta$ signaling pathways responsible for the maintenance of cardiovascular homeostasis.

Other possible loci are currently under investigation [7] (Table 1).

Patients are separated into three major subtypes of HHT based on the affected gene, HHT1, HHT2, and juvenile polyposis-HHT overlap syndrome. HHT1 patients have a mutation in the ENG gene encoding the protein endoglin, a coreceptor for type I and II TGF- $\beta$ pathways. HHT2 patients have a mutation in the ACLVR1 (activin receptor-like kinase 1 ) gene encoding ALK1, a type-1 receptor in the TGF- $\beta$ superfamily. Mutations in these two genes account for $~ 99 \%$ of cases of HHT. The different HHT classification reflects differing natural histories, with HHT1 patients experiencing more severe GI bleeds and more frequent pulmonary arterial hypertension [8]. 1\% - 2\% of HHT patients have a mutation in MADH4 that results in juvenile polyposis-HHT overlap syndrome [9]. MADH4 encodes Smad-4, a downstream transcription factor of ALK1. The remaining cases of HHT attributed to an identified gene account for $<1 \%$ of total cases and are caused by mutations in GDF2/BMP9, a TFG- $\beta$ ligand, and BMPR2, a type 2 TGF- $\beta$ receptor [1] [6] [10].

An unreported missense mutation in the exon 7 of ACVRL1 resulting in a p. Ser333Asn amino acid alteration is the suspected cause of this patient's late-onset HHT (Table 2).

ALK1 acts in endothelial cells, where it functions as a regulator of the activation stage of angiogenesis, promoting endothelial cell proliferation and migration. TGF- $\beta$ ligands, including BMP9, bind to a Type-2 TGF- $\beta$ receptor, resulting in the phosphorylation of ALK1, or other Type-1 TGF- $\beta$ receptor receptors. ALK1 subsequently phosphorylates Smad-1/5/8, causing dimerization with Smad-4 [11]. The Smad dimer then enters the nucleus where it acts as a transcription factor, regulating the transcription of angiogenesis genes such as VEGF. Reduced presence of endoglin, a coreceptor for ALK family of receptors, has been shown to decrease the activity of the ALK1 pathway, as well as the ALK5 pathway that counterbalances ALK1 activity by promoting cell 
Table 1. Summary of genes causing HHT [6] [14].

\begin{tabular}{|c|c|c|c|c|}
\hline Type & Gene/protein & Gene description & Mutation types & Mutation locations \\
\hline HHT1 & ENG/Endoglin & $\begin{array}{l}\text { Member of TGF } \beta \text { receptor } \\
\text { complex }\end{array}$ & $\begin{array}{l}\text { Point mutations; } \\
\text { duplications; } \\
\text { deletions; } \\
\text { insertions }\end{array}$ & Exons 1 - 14; \\
\hline HНT2 & ACVRL1/ALK1 & $\begin{array}{c}\text { Type } 1 \text { cell surface receptor in TGF } \beta \\
\text { superfamily }\end{array}$ & $\begin{array}{l}\text { Point mutations; } \\
\text { duplications; } \\
\text { deletions; } \\
\text { insertions }\end{array}$ & $\begin{array}{l}\text { Exons } 2 \text { - 10; } \\
\text { Introns } 3 \text { - } 9\end{array}$ \\
\hline $\begin{array}{l}\text { HHT associated with } \\
\text { Juvenile Polyposis }\end{array}$ & MADH4/Smad4 & Transcription factor in TGF $\beta$ & $\begin{array}{c}\text { Point mutations; } \\
\text { duplications; } \\
\text { deletions; insertions }\end{array}$ & Exons 4, 7, $10-13$ \\
\hline HHT-5 & BMP9 (GDF2)/BMP9 & $\begin{array}{l}\text { TGF } \beta \\
\text { ligand }\end{array}$ & Point mutations & Exons 1, 2 \\
\hline
\end{tabular}

Table 2. Documented predicted pathogenic ACLVR-1 mutations according to the University of Utah HHT mutation database.

\begin{tabular}{|c|c|c|c|}
\hline Nucleotide change & Mutation type & Protein change & Reference \\
\hline c.? & Deletion & p.0 & {$[15]$} \\
\hline c. $24 \mathrm{~A}>\mathrm{T}$ & Missense & p.Lys8Ans & {$[16]$} \\
\hline c.31_50del20 & Deletion & p.Leu11Glyfs*20 & {$[17]$} \\
\hline c.37delC & Deletion & p.Leu13Cysfs*2 & {$[18]$} \\
\hline c.50dupT & Duplication & p.Leu17Phefs*21 & [19] \\
\hline c.50_53delTGGT & Deletion & p.Leu*17 & {$[20]$} \\
\hline c. $61+1 \mathrm{G}>\mathrm{A}$ & Missense & p.? & [14] \\
\hline c. $61+10 \mathrm{G}>\mathrm{A}$ & Splice Site & p.? & {$[21]$} \\
\hline c.65delA & Deletion & p.Asp22Alafs*3 & [14] \\
\hline c.69delT & Deletion & p.Val24* & [14] \\
\hline c.74_78delAGCCGins176bp & Delins & p.? & [19] \\
\hline c.81dupT & Duplication & p.Arg28Serfs*10 & {$[22]$} \\
\hline c.86delG & Deletion & p.Gly29Alafs*4 & [23] \\
\hline c. $88 \mathrm{C}>\mathrm{T}$ & Missense & p.Pro30Ser & {$[24]$} \\
\hline c. $95 \mathrm{~T}>\mathrm{G}$ & Missense & p.Val32Gly & [25] \\
\hline c.100dupT & Duplication & p.Cys34Leufs*4 & [19] \\
\hline c.100_115del16 & Deletion & p.Cys34Hisfs*15 & {$[26]$} \\
\hline c. $101 \mathrm{G}>\mathrm{A}$ & Missense & p.Cys34Tyr & [24] \\
\hline c. $102 \mathrm{C}>\mathrm{A}$ & Missense & p.Cys34* & [25] \\
\hline c. $106 \mathrm{~T}>\mathrm{C}$ & Missense & p.Cys36Arg & {$[14]$} \\
\hline c. $107 \mathrm{G}>\mathrm{A}$ & Missense & p.Cys36Tyr & [27] \\
\hline c.115_118dupCCAC & Duplication & p.His40Profs*130 & {$[28]$} \\
\hline c. $121 \mathrm{~T}>\mathrm{C}$ & Missense & p.Cys41Arg & [28] \\
\hline c.128_132delGGCCT & Deletion & p.Gly43Aspfs*124 & [29] \\
\hline c.129delG & Deletion & p.Pro44Leufs*10 & {$[14]$} \\
\hline c.136_137delTGinsCT & Delins & p.Cys46Leu & [29] \\
\hline c. $138 \mathrm{C}>\mathrm{A}$ & Missense & p.Cys46* & {$[19]$} \\
\hline c.139_140insCG & Insertion & p.Arg47Profs*8 & {$[30]$} \\
\hline c.139dupC & Duplication & p.Arg47Profs*122 & {$[24]$} \\
\hline
\end{tabular}




\section{Continued}

$\begin{array}{lll}\text { c.140G }>\text { C } & \text { Missense } & \text { p.Arg47Pro } \\ \text { c.142G }>\text { A } & \text { Missense } & \text { p.Gly48Arg } \\ \text { c.143G }>\text { A } & \text { Missense } & \text { p.Gly48Glu }\end{array}$

c.143_147delGGGCCinsAGCCT

Delins

c.145dupG

c.145delG

Duplication

c.147delC

Deletion

p.Gly48_Ala49delinsGluPro

c. $148 \mathrm{~T}>\mathrm{G}$

Deletion

c.149G $>$ A

Missense

c. $150 \mathrm{G}>\mathrm{T}$

Missense

c. $150 \mathrm{G}>\mathrm{A}$

Missense

p.Ala49Glyfs*120

p.Ala49Profs*5

p.Trp50Glyfs*4

p.Trp50Gly

p.Trp50*

p.Trp50Cys

Missense

p.Trp50*

c. $152 \mathrm{G}>\mathrm{A}$

Missense

p.Cys51Tyr

c. $154 \mathrm{~A}>\mathrm{G}$

Missense

c.155delC

p.Thr52Ala

Deletion

p.Thr52Lysfs*2

c.164_169delTGGTGC

Deletion

c. $172 \mathrm{G}>\mathrm{T}$

Missense

c.183delG

c.190delC

Deletion

Deletion

c.191delA

Deletion

c.193G $>$ T

Missense

c.197A > C

Missense

c.199C $>$ T

Missense

p.Leu55_Val56del

p.Glu58*

p.Arg61Serfs*61

p.Gln64Argfs*58

p.Gln64Argfs*58

p.Glu65*

p.His66Pro

c. $200 \mathrm{G}>\mathrm{A}$

p.Arg67Trp

Missense

p.Arg67Gln

Missense

c. $202 \mathrm{G}>\mathrm{T}$

p.Gly68Cys

Duplication

c.203delG

Deletion

p.Cys69Leufs*100

p.Gly68Alafs*54

Duplication

p.Asn71Alafs*53

Missense

c. $205 \mathrm{~T}>\mathrm{C}$

Missense

p.Cys69Arg

c.206G $>$ T

p.Cys69Phe

Missense

p.Cys69Tyr

Missense

Deletion

c.214_219delTTGCAC

p.Gly70Arg

p.Leu72_His73del

c.215delT

c.218_219insAA

Deletion

Insertion

p.Leu72Cysfs*50

p.His73Glnfs*50

Deletion

c. $230 \mathrm{G}>\mathrm{A}$

Missense

p.Cys77_Arg80del

p.Cys77Tyr

c. $231 \mathrm{C}>\mathrm{G}$

Missense

p.Cys77Trp

Missense

p.Gly79Arg

[40]

Duplication

p.Arg80Alafs*89

[23]

Duplication

p.Thr82Hisfs $* 87$

Deletion

p.Thr82del

[27]

Deletion

p.Glu83Serfs*39

Duplication

p.Val85Argfs*84

[42] 


\section{Continued}

\begin{tabular}{|c|c|c|c|}
\hline c. $259 \mathrm{C}>\mathrm{G}$ & Missense & p.His87Asp & [19] \\
\hline c. $263 \mathrm{~A}>\mathrm{G}$ & Missense & p.Tyr88Cys & [17] \\
\hline c. $265 \mathrm{~T}>\mathrm{C}$ & Missense & p.Cys89Arg & [14] \\
\hline c. $266 \mathrm{G}>\mathrm{A}$ & Missense & p.Cys89Tyr & [20] \\
\hline c. $269 \mathrm{G}>\mathrm{A}$ & Missense & p.Cys90Tyr & [25] \\
\hline c. $270 \mathrm{C}>\mathrm{G}$ & Missense & p.Cys90Trp & [43] \\
\hline c. $270 \mathrm{C}>\mathrm{A}$ & Missense & p.Cys90* & [44] \\
\hline c. $283 \mathrm{~T}>\mathrm{C}$ & Missense & p.Cys95Arg & [28] \\
\hline c. $286 \mathrm{~A}>\mathrm{G}$ & Missense & p.Asn96Asp & [34] \\
\hline c. $287 \mathrm{~A}>\mathrm{G}$ & Missense & p.Asn96Ser & [45] \\
\hline c.289_295delCACAACG & Deletion & p.His97Cysfs*23 & [14] \\
\hline c.289_294delCACAAC & Deletion & p.His97_Ans98del & [35] \\
\hline c. $293 \mathrm{~A}>\mathrm{G}$ & Missense & p.Asn98Ser & [46] \\
\hline c.301_307delCTGGTGC & Deletion & p.Leu101Trpfs*19 & [31] \\
\hline c. $313+1 \mathrm{G}>\mathrm{A}$ & Missense & p.? & [14] \\
\hline c. $314-3 C>G$ & Splice Site & p.? & [46] \\
\hline c.319delC & Deletion & p.Gln107Asnfs*15 & [37] \\
\hline c.321delA & Deletion & p.Gln107Hisfs*15 & [40] \\
\hline c. $329 \mathrm{C}>\mathrm{A}$ & Missense & p.Ser110* & [14] \\
\hline c. $334 \mathrm{C}>\mathrm{T}$ & Missense & p.Gln112* & [14] \\
\hline c. $352 \mathrm{C}>\mathrm{T}$ & Missense & p.Gln118* & [39] \\
\hline c.353_360dupAGCTGGCC & Duplication & p.Leu121Serfs*4 & [47] \\
\hline c.374_375dupCC & Duplication & p.Val126Profs $* 40$ & [39] \\
\hline c. $383 \mathrm{C}>\mathrm{A}$ & Missense & p.Ala128Asp & [48] \\
\hline c.400delG & Deletion & p.Ala134Profs*31 & [34] \\
\hline c.406_409delGGTG & Deletion & p.Gly136Serfs*28 & [34] \\
\hline c. $423 \mathrm{G}>\mathrm{A}$ & Missense & p.Trp141* & [38] \\
\hline c. $430 \mathrm{C}>\mathrm{T}$ & Missense & p.Arg144* & [23] \\
\hline c.435dupG & Duplication & p.Arg146Glufs*23 & [24] \\
\hline c.435delG & Deletion & p.Arg146Glyfs*19 & [49] \\
\hline c. $439 \mathrm{C}>\mathrm{T}$ & Missense & p.Gln147* & [39] \\
\hline c. $448 \mathrm{C}>\mathrm{T}$ & Missense & p.Gln $150 *$ & [28] \\
\hline c. $474 \mathrm{~A}>\mathrm{T}$ & Missense & p.Gly158Gly & [24] \\
\hline c. $475 \mathrm{G}>\mathrm{T}$ & Missense & p.Glu159* & [38] \\
\hline c.476_477delAG & Deletion & p.Glu159Valfs*9 & [20] \\
\hline c.480_486dupCAGTCTC & Duplication & p.Ile163Glnfs*8 & [43] \\
\hline c.480_481delCA & Deletion & p.Leu162Hisfs*6 & [26] \\
\hline c. $481 \mathrm{~A}>\mathrm{G}$ & Missense & p.Ser161Gly & [19] \\
\hline c. $505 \mathrm{C}>\mathrm{T}$ & Missense & p.Gln169* & [43] \\
\hline c.510delC & Deletion & p.Asp171Thrfs*87 & [31] \\
\hline c.525_525 + 1delGG & Deletion & p.Asp176Thrfs*82 & [25] \\
\hline c. $525+1 \mathrm{G}>\mathrm{C}$ & Splice Site & p.? & [43] \\
\hline c. $525+1 \mathrm{G}>\mathrm{A}$ & Splice Site & p.? & [17] \\
\hline
\end{tabular}




\section{Continued}

\begin{tabular}{|c|c|c|c|}
\hline c.525 + 1delG & Deletion & p.? & [19] \\
\hline c. $525+3 A>G$ & Splice Site & p.? & [17] \\
\hline c. $525+3 \mathrm{~A}>\mathrm{T}$ & Splice Site & p.? & {$[50]$} \\
\hline c.526-7C > G & Splice Site & p.? & {$[37]$} \\
\hline c.526-3C > G & Splice Site & p.? & {$[30]$} \\
\hline c.526-1G > A & Missense & p.? & [14] \\
\hline c. $526 \mathrm{G}>\mathrm{T}$ & Missense & p.Asp176Tyr & [37] \\
\hline c.526delG & Deletion & p.Asp176Thrfs*82 & [19] \\
\hline c.536A > C & Missense & p.Asp179Ala & {$[51]$} \\
\hline c.540_541insA & Insertion & p.Asp181Argfs*44 & [29] \\
\hline c.563delC & Deletion & p.Ser188* & [19] \\
\hline c.567delG & Deletion & p.Leu190Serfs*68 & [47] \\
\hline c.573delC & Deletion & p.Phe192Serfs*66 & {$[26]$} \\
\hline c. $590 \mathrm{C}>\mathrm{T}$ & Missense & p.Thr197Ile & [24] \\
\hline c.593T > A & Missense & p.Val198Glu & {$[52]$} \\
\hline c. $598 \mathrm{C}>\mathrm{G}$ & Missense & p.Arg200Gly & [43] \\
\hline c. $601 \mathrm{C}>\mathrm{T}$ & Missense & p.Gln201* & {$[45]$} \\
\hline c. $601 \mathrm{C}>\mathrm{A}$ & Missense & p.Gln201Lys & [30] \\
\hline c. $602 \mathrm{~A}>\mathrm{G}$ & Missense & p.Gln201Arg & {$[53]$} \\
\hline c. $602 \mathrm{~A}>\mathrm{C}$ & Missense & p.Gln201Pro & [19] \\
\hline c. $611 \mathrm{~T}>\mathrm{G}$ & Missense & p.Leu204Trp & [19] \\
\hline c. $614 \mathrm{~T}>\mathrm{G}$ & Missense & p.Val205Gly & [43] \\
\hline c. $617 \mathrm{~A}>\mathrm{G}$ & Missense & p.Glu206Gly & {$[20]$} \\
\hline c.617_625delAGTGTGTGG & Deletion & p.Glu206_Val208del & {$[54]$} \\
\hline c.620delG & Deletion & p.Cys207Leufs*51 & {$[14]$} \\
\hline c.623_624dupTG & Duplication & p.Gly209Trpfs*50 & [39] \\
\hline c.626-9_629del13 & Deletion & p.? & {$[37]$} \\
\hline c.626-5_634del14 & Deletion & p.? & [39] \\
\hline c. $626-3 \mathrm{C}>\mathrm{G}$ & Splice Site & p.? & {$[55]$} \\
\hline c. $632 \mathrm{G}>\mathrm{A}$ & Missense & p.Gly211Asp & {$[51]$} \\
\hline с. $639 \mathrm{~T}>\mathrm{G}$ & Missense & p.Tyr213* & [14] \\
\hline c.641delG & Deletion & p.Gly214Alafs*44 & [45] \\
\hline c. $643 \mathrm{G}>\mathrm{A}$ & Missense & p.Glu215Lys & {$[31]$} \\
\hline c. $647 \mathrm{~T}>\mathrm{G}$ & Missense & p.Val216Gly & [19] \\
\hline c. $649 \mathrm{~T}>\mathrm{G}$ & Missense & p.Trp217Gly & [45] \\
\hline c. $650 \mathrm{G}>\mathrm{A}$ & Missense & p.Trp217* & {$[20]$} \\
\hline c. $651 \mathrm{G}>\mathrm{A}$ & Missense & p.Trp217* & {$[24]$} \\
\hline c.653_654delGGinsCC & Delins & p.Arg218Pro & {$[52]$} \\
\hline c. $656 \mathrm{G}>\mathrm{A}$ & Missense & p.Gly219Asp & {$[24]$} \\
\hline c. $661 \mathrm{~T}>\mathrm{G}$ & Missense & p.Trp221Gly & {$[14]$} \\
\hline c. $662 \mathrm{G}>\mathrm{A}$ & Missense & p.Trp221* & [19] \\
\hline c. $663 \mathrm{G}>\mathrm{A}$ & Missense & p.Trp221* & {$[47]$} \\
\hline c.664_668delCACGG & Deletion & p.His222* & [31] \\
\hline
\end{tabular}




\section{Continued}

\begin{tabular}{|c|c|c|c|}
\hline c. $667 \mathrm{G}>\mathrm{C}$ & Missense & p.Gly223Arg & [31] \\
\hline c. $670 \mathrm{G}>\mathrm{A}$ & Missense & p.Glu224Lys & [25] \\
\hline c. $673 \mathrm{~A}>\mathrm{T}$ & Missense & p.Ser225Cys & [16] \\
\hline c.673_674delAG & Deletion & p.Ser225Cysfs*11 & [47] \\
\hline c. $676 \mathrm{G}>\mathrm{C}$ & Missense & p.Val226Leu & [30] \\
\hline c. $677 \mathrm{~T}>\mathrm{A}$ & Missense & p.Val226Glu & [45] \\
\hline c.682delG & Deletion & p.Val228Serfs*30 & [31] \\
\hline c. $683 \mathrm{~T}>\mathrm{A}$ & Missense & p.Val228Asp & [19] \\
\hline c. $686 \mathrm{~A}>\mathrm{T}$ & Missense & p.Lys229Met & [55] \\
\hline c. $686 \mathrm{~A}>\mathrm{G}$ & Missense & p.Lys229Arg & [31] \\
\hline c.696_698delCTC & Deletion & p.Ser233del & [56] \\
\hline c. $698 \mathrm{C}>\mathrm{T}$ & Missense & p.Ser233Leu & [37] \\
\hline c. $698 \mathrm{C}>\mathrm{A}$ & Missense & p.Ser233* & [57] \\
\hline c.704delA & Deletion & p.Asp235Valfs*23 & [31] \\
\hline c.709C > T & Missense & p.Gln237* & [25] \\
\hline c. $716 \mathrm{G}>\mathrm{A}$ & Missense & p.Trp239* & [46] \\
\hline c.743_744delCA & Deletion & p.Thr248Serfs*143 & [29] \\
\hline c.760_762delGAC & Deletion & p.Asp254del & [18] \\
\hline c.759_761delCGA & Deletion & p.Asp254del & [31] \\
\hline c.772 + 3_772 + 4dupAA & Duplication & p.? & [43] \\
\hline c. $772+5 \mathrm{G}>\mathrm{A}$ & Missense & p.? & [14] \\
\hline c. $772+21 \mathrm{~T}>\mathrm{A}$ & Splice Site & p.? & [25] \\
\hline c. $773-2 \mathrm{~A}>\mathrm{G}$ & Splice Site & p.? & [31] \\
\hline c. $773-2 \mathrm{~A}>\mathrm{C}$ & Splice Site & p.? & [19] \\
\hline c. $778 \mathrm{~A}>\mathrm{C}$ & Missense & p.Ile260Leu & [24] \\
\hline c. $793 \mathrm{~A}>\mathrm{C}$ & Missense & p.Thr265Pro & [37] \\
\hline c.811_823del13bp & Deletion & p.Thr271Serfs*26 & [35] \\
\hline c. $818 \mathrm{~T}>\mathrm{C}$ & Missense & p.Leu273Pro & [48] \\
\hline c.821_824dupGGCT & Duplication & p.Ile276Alafs*117 & [40] \\
\hline c. $822 \mathrm{G}>\mathrm{A}$ & Missense & p.Trp274* & [45] \\
\hline c. $827 \mathrm{~T}>\mathrm{C}$ & Missense & p.Ile276Thr & [48] \\
\hline c.835_837dupTAC & Duplication & p.Tyr279dup & [14] \\
\hline c. $838 \mathrm{C}>\mathrm{G}$ & Missense & p.His280Asp & [25] \\
\hline с. $839 \mathrm{~A}>\mathrm{G}$ & Missense & p.His280Arg & [45] \\
\hline c.842delA & Deletion & p.Glu281Glyfs*20 & [26] \\
\hline c. $851 \mathrm{C}>\mathrm{T}$ & Missense & p.Ser284Phe & [48] \\
\hline c.853dupC & Duplication & p.Leu285Profs*107 & [20] \\
\hline c. $853 \mathrm{C}>\mathrm{T}$ & Missense & p.Leu285Phe & [31] \\
\hline c. $854 \mathrm{~T}>\mathrm{C}$ & Missense & p.Leu285Pro & [58] \\
\hline c. $858 \mathrm{C}>\mathrm{G}$ & Missense & p.Tyr286* & [25] \\
\hline c. $858 \mathrm{C}>\mathrm{A}$ & Missense & p.Tyr286* & [35] \\
\hline c.863_909del47 & Deletion & p.Phe288Cysfs*88 & [14] \\
\hline c.864dupT & Duplication & p.Leu289Serfs*103 & [38] \\
\hline
\end{tabular}




\section{Continued}

\begin{tabular}{|c|c|c|c|}
\hline c. $866 \mathrm{~T}>\mathrm{C}$ & Missense & p.Leu289Pro & [24] \\
\hline c.870delG & Deletion & p.Arg219Aspfs*10 & [14] \\
\hline c.874delC & Deletion & p.Gln292Argfs*9 & [30] \\
\hline c. $874 \mathrm{C}>\mathrm{T}$ & Missense & p.Gln292* & [19] \\
\hline c. $875 \mathrm{~A}>\mathrm{C}$ & Missense & p.Gln292Pro & [26] \\
\hline c. $881 \mathrm{~T}>\mathrm{G}$ & Missense & p.Leu294Arg & [45] \\
\hline c. $905 \mathrm{~T}>\mathrm{G}$ & Missense & p.Leu302Arg & [26] \\
\hline c. $.913 \mathrm{~T}>\mathrm{C}$ & Missense & p.Ser305Pro & [48] \\
\hline c.913delT & Deletion & p.Ser305Profs $* 49$ & [59] \\
\hline c. $914 \mathrm{C}>\mathrm{T}$ & Missense & p.Ser305Phe & [17] \\
\hline c. $916 \mathrm{G}>\mathrm{C}$ & Missense & p.Ala306Pro & [31] \\
\hline c. $917 \mathrm{C}>\mathrm{A}$ & Missense & p.Ala306Glu & [30] \\
\hline c.921_927dupATGCGGC & Duplication & p.Leu310Metfs*84 & [47] \\
\hline c. $924 \mathrm{C}>\mathrm{A}$ & Missense & p.Cys308* & [38] \\
\hline c. $925 \mathrm{G}>\mathrm{T}$ & Missense & p.Gly309Cys & [25] \\
\hline c. $925 \mathrm{G}>\mathrm{A}$ & Missense & p.Gly309Ser & [39] \\
\hline c. $936 \mathrm{C}>\mathrm{G}$ & Missense & p.His312Gln & [60] \\
\hline c. $940 \mathrm{C}>\mathrm{T}$ & Missense & p.His314Tyr & [31] \\
\hline c.941_951del11 & Deletion & p.His314Leufs*74 & [30] \\
\hline c.950T > C & Missense & p.Ile317Thr & [14] \\
\hline c. $956 \mathrm{G}>\mathrm{A}$ & Missense & p.Gly319Asp & [25] \\
\hline c. $961 \mathrm{C}>\mathrm{T}$ & Missense & p.Gln321* & [39] \\
\hline c.969dupA & Duplication & p.Pro324Thrfs*68 & [43] \\
\hline c.972delA & Deletion & p.Ala325Profs*29 & [31] \\
\hline c. $976 \mathrm{~A}>\mathrm{G}$ & Missense & p.Ile326Val & [59] \\
\hline c. $982 \mathrm{C}>\mathrm{T}$ & Missense & p.His328Tyr & [17] \\
\hline c. $983 \mathrm{~A}>\mathrm{C}$ & Missense & p.His328Pro & [27] \\
\hline c. $985 \mathrm{C}>\mathrm{T}$ & Missense & p.Arg329Cys & [14] \\
\hline c. $986 \mathrm{G}>\mathrm{A}$ & Missense & p.Arg329His & [23] \\
\hline c. $988 \mathrm{G}>\mathrm{T}$ & Missense & p.Asp330Tyr & [35] \\
\hline c. $988 \mathrm{G}>\mathrm{C}$ & Missense & p.Asp330His & [30] \\
\hline c. $988 \mathrm{G}>\mathrm{A}$ & Missense & p.Asp330Asn & [39] \\
\hline C.991_1044del54bp & Missense & p.Phe331_Asp348del54bp & [30] \\
\hline c.992T > C & Missense & p.Phe331Ser & [24] \\
\hline c.994_995insGACTTA & Insertion & p.Phe331_Lys332insArgLeu & [14] \\
\hline c. $997 \mathrm{~A}>\mathrm{G}$ & Missense & p.Ser333Gly & [26] \\
\hline c. $998 \mathrm{G}>\mathrm{T}$ & Missense & p.Ser333Ile & [38] \\
\hline c. $998 \mathrm{G}>\mathrm{A}$ & Missense & p.Ser333Asn & [14] \\
\hline c.1000delC & Deletion & p.Arg334Alafs*20 & [54] \\
\hline c.1000_1005delCGCAATinsG & Delins & p.Arg334Glyfs*56 & [31] \\
\hline c. $1003 \mathrm{~A}>\mathrm{C}$ & Missense & p.Asn335His & [45] \\
\hline c.1010dupT & Duplication & p.Val338Glyfs*54 & [14] \\
\hline c. $1010 \mathrm{~T}>\mathrm{C}$ & Missense & p.Leu337Pro & [31] \\
\hline
\end{tabular}




\section{Continued}

\begin{tabular}{|c|c|c|c|}
\hline c.1010delT & Deletion & p.Leu337Argfs*17 & [25] \\
\hline c. $1013 \mathrm{~T}>\mathrm{A}$ & Missense & p.Val338Asp & [30] \\
\hline c. $1022 \mathrm{~A}>\mathrm{T}$ & Missense & p.Asn341Ile & [30] \\
\hline c. $1023 \mathrm{C}>\mathrm{G}$ & Missense & p.Asn341Lys & [31] \\
\hline c. $1027 \mathrm{C}>\mathrm{T}$ & Missense & p.Gln343* & [14] \\
\hline c. $1030 \mathrm{~T}>\mathrm{C}$ & Missense & p.Cys344Arg & [61] \\
\hline c. $1031 \mathrm{G}>\mathrm{T}$ & Missense & p.Cys344Phe & [31] \\
\hline c.1031G > A & Missense & p.Cys344Tyr & [33] \\
\hline c.1039G > C & Missense & p.Ala347Pro & [31] \\
\hline c. $1040 \mathrm{C}>\mathrm{A}$ & Missense & p.Ala347Asp & [45] \\
\hline c.1040_1042delCCG & Deletion & p.Ala347del & [62] \\
\hline c.1042delG & Deletion & p.Asp348Thrsf*6 & [39] \\
\hline c.1043_1048 + 1dupACCTGGG & Insertion & p.? & [14] \\
\hline c. $1046 \mathrm{~T}>\mathrm{C}$ & Missense & p.Leu349Pro & [45] \\
\hline c.1048G > C & Missense & p.Gly350Arg & [39] \\
\hline c. $1048 \mathrm{G}>\mathrm{A}$ & Missense & p.Gly350Ser & [26] \\
\hline c. $1048+1 G>C$ & Missense & p.? & [14] \\
\hline c. $1048+1 G>A$ & Splice Site & p.? & [25] \\
\hline c. $1048+5 G>T$ & Splice Site & p.? & [47] \\
\hline c. $1048+5 G>A$ & Missense & p.? & [14] \\
\hline c.1049-4_1049-2delACAinsCC & Delins & p.? & [28] \\
\hline c. $1054 \mathrm{G}>\mathrm{C}$ & Missense & p.Ala352Pro & [35] \\
\hline c. $1055 \mathrm{C}>\mathrm{A}$ & Missense & p.Ala352Asp & [46] \\
\hline c. $1061 \mathrm{~T}>\mathrm{A}$ & Missense & p.Met354Lys & [29] \\
\hline c.1061_1068delTGCACTCA & Deletion & p.Met354Thrfs*35 & [39] \\
\hline c.1062_1080dup19 & Duplication & p.Try361Alafs*37 & [40] \\
\hline c.1069C > T & Missense & p.Gln357* & [48] \\
\hline c.1073delG & Deletion & p.Gly358Alafs*57 & [39] \\
\hline c. $1083 \mathrm{C}>\mathrm{A}$ & Missense & p.Tyr361* & [14] \\
\hline c.1102_1105delCCGA & Deletion & p.Pro368Glufs*46 & [14] \\
\hline c.1107_1108delAG & Deletion & p.Arg369Serfs*22 & [39] \\
\hline c. $1111 \mathrm{G}>\mathrm{A}$ & Missense & p.Gly371Ser & [19] \\
\hline c.1112dupG & Duplication & p.Thr372Hisfs $* 20$ & [23] \\
\hline c. $1115 \mathrm{C}>\mathrm{T}$ & Missense & p.Thr372Ile & [28] \\
\hline c.1118delA & Deletion & p.Lys373Serfs*42 & [19] \\
\hline c. $1120 \mathrm{C}>\mathrm{T}$ & Missense & p.Arg374Trp & [38] \\
\hline c.1120_1137del18 & Deletion & p.Arg374_Glu379del & [39] \\
\hline c. $1121 \mathrm{G}>\mathrm{A}$ & Missense & p.Arg374GIn & [23] \\
\hline c.1122_1125dupGTAC & Duplication & p.Met376Valfs*17 & [31] \\
\hline c. $1123 \mathrm{~T}>\mathrm{C}$ & Missense & p.Tyr375His & [23] \\
\hline c. $1124 \mathrm{~A}>\mathrm{G}$ & Missense & p.Tyr375Cys & [52] \\
\hline c. $1126 \mathrm{~A}>\mathrm{G}$ & Missense & p.Met376Val & [31] \\
\hline с. $1127 \mathrm{~T}>\mathrm{G}$ & Missense & p.Met376Arg & [56] \\
\hline
\end{tabular}




\section{Continued}

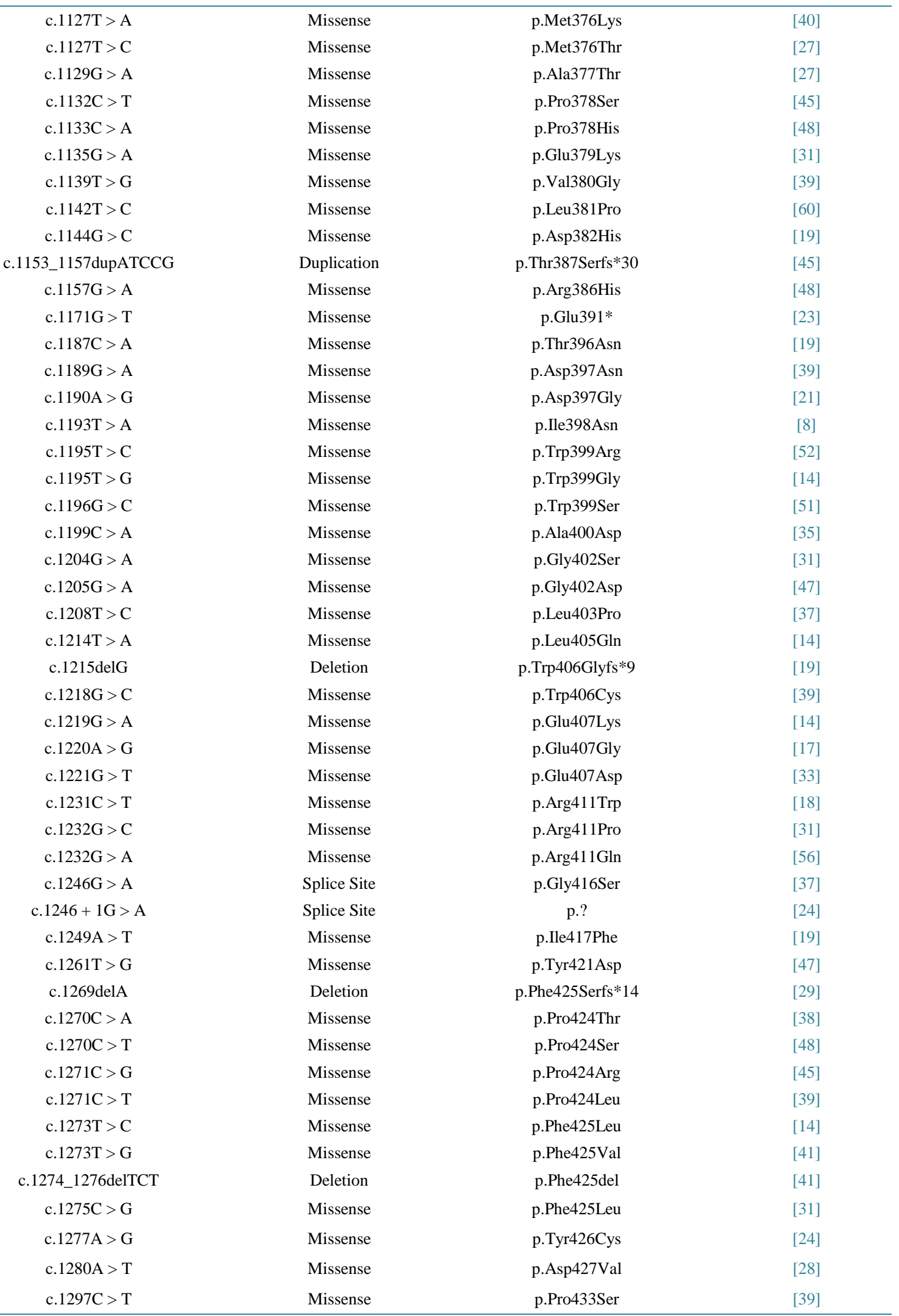




\section{Continued}

\begin{tabular}{|c|c|c|c|}
\hline c. $1298 \mathrm{C}>\mathrm{G}$ & Missense & p.Pro433Arg & [24] \\
\hline c.1299delC & Deletion & p.Ser434Alafs*5 & [23] \\
\hline c.1302_1303delCTinsA & Delins & p.Ser434Argfs*5 & [37] \\
\hline c.1309delG & Deletion & p.Asp437Thrfs*2 & [19] \\
\hline c. $1310 A>G$ & Missense & p.Asp437Gly & [19] \\
\hline c. $1313 \mathrm{~T}>\mathrm{C}$ & Missense & p.Met438Thr & [39] \\
\hline c. $1313 \mathrm{~T}>\mathrm{G}$ & Missense & p.Met438Arg & [19] \\
\hline c. $1315 \mathrm{~A}>\mathrm{T}$ & Missense & p.Lys439* & [14] \\
\hline c.1318_1320delAAG & Deletion & p.Lys440del & [24] \\
\hline c. $1321 \mathrm{G}>\mathrm{A}$ & Missense & p.Val441Met & [48] \\
\hline c.1324_1326delGTG & Deletion & p.Val442del & [19] \\
\hline c. $1324 \mathrm{G}>\mathrm{A}$ & Missense & p.Val442Met & [53] \\
\hline c.1325T > C & Missense & p.Val442Ala & [57] \\
\hline c.1331_1332dupTG & Duplication & p.Asp445Trpfs*21 & [14] \\
\hline c. $1336 \mathrm{C}>\mathrm{T}$ & Missense & p.Gln446* & [19] \\
\hline c. $1345 \mathrm{C}>\mathrm{A}$ & Missense & p.Pro449Thr & [47] \\
\hline c. $1345 \mathrm{C}>\mathrm{T}$ & Missense & p.Pro449Ser & [45] \\
\hline c. $1346 \mathrm{C}>\mathrm{T}$ & Missense & p.Pro449Leu & [29] \\
\hline c.1347dupC & Duplication & p.Thr450Hisfs*4 & [14] \\
\hline c.1354_1355delCC & Deletion & p.Pro452* & [14] \\
\hline c. $1355 \mathrm{C}>\mathrm{T}$ & Missense & p.Pro452Leu & [48] \\
\hline c. $1377+1 \mathrm{G}>\mathrm{A}$ & Splice Site & p.? & [41] \\
\hline c. $1378-2 A>G$ & Splice Site & p.? & [29] \\
\hline c. $1378-1 \mathrm{G}>\mathrm{T}$ & Splice Site & p.? & [47] \\
\hline c. $1385 \mathrm{C}>\mathrm{G}$ & Missense & p.Ser462* & [63] \\
\hline c.1388delG & Deletion & p.Gly463Alafs*2 & [53] \\
\hline c.1390delC & Deletion & p.Leu464* & [64] \\
\hline c. $1396 \mathrm{C}>\mathrm{T}$ & Missense & p.Gln466* & [19] \\
\hline c. $1408 \mathrm{G}>\mathrm{T}$ & Missense & p.Glu470* & [39] \\
\hline c. $1413 \mathrm{C}>\mathrm{A}$ & Missense & p.Cys471* & [24] \\
\hline c.1428dupC & Duplication & p.Ser477Leufs*17 & [31] \\
\hline c. $1433 \mathrm{C}>\mathrm{A}$ & Missense & p.Ala478Asp & [58] \\
\hline c. $1435 \mathrm{C}>\mathrm{T}$ & Missense & p.Arg479* & [63] \\
\hline c. $1436 \mathrm{G}>\mathrm{A}$ & Missense & p.Arg479Gln & [61] \\
\hline c. $1436 G$ > C & Missense & p.Arg479Pro & [45] \\
\hline c. $1436 \mathrm{G}>\mathrm{T}$ & Missense & p.Arg479Leu & [31] \\
\hline c. $1438 \mathrm{C}>\mathrm{T}$ & Missense & p.Leu480Phe & [20] \\
\hline c. $1450 \mathrm{C}>\mathrm{G}$ & Missense & p.Arg484Gly & [65] \\
\hline c. $1450 \mathrm{C}>\mathrm{T}$ & Missense & p.Arg484Trp & [18] \\
\hline c.1450delCinsTG & Delins & p.Arg484Trpfs*10 & [63] \\
\hline с. $1451 \mathrm{G}>\mathrm{A}$ & Missense & p.Arg484Gln & [20] \\
\hline c. $1451 \mathrm{G}>\mathrm{T}$ & Missense & p.Arg484Leu & [57] \\
\hline c. $1460 \mathrm{~A}>\mathrm{C}$ & Missense & p.Lys487Thr & [51] \\
\hline c. $1468 \mathrm{C}>\mathrm{T}$ & Missense & p.Gln490* & [18] \\
\hline c. $1475 \mathrm{~T}>\mathrm{A}$ & Missense & p.Ile492Asn & [8] \\
\hline
\end{tabular}


Quiescence [12]. In healthy individuals, increases in endoglin levels can be seen after episodes of vascular damage. Circulating endothelial cells of HHT patients display a decreased level of endoglin and dysfunctional ALK1 and ALK5 signaling pathways. Recent data suggests ALK1 may also play a role in regulating the resolution phase of angiogenesis, further expanding the mechanism behind HHT dysgenesis [13].

\section{Conclusion}

Here we have a late-onset variation of HHT in the presence of a novel, suspected pathogenic mutation in ACVRL1. HHT patients typically present with recurrent nosebleeds by the age of 30 (90\%), superficial telangiectasias by the age of 40 (67\%) and GI bleeding starting in their 50s. This patient reported recurrent epistaxis onset in her late middle age and at the age of 62 did not complain of GI bleeds. Abdomen, pelvis and chest CT revealed an absence of visceral AVMs. A mutation occurring at the same nucleotide position, c.998, but resulting in a different amino acid change, has been found in multiple other HHT patients. The clinical presentation of these patients is unknown; however the presentation of our patient suggests that a c.998G > A missense mutation causes a late-onset HHT clinical presentation manageable with supportive care.

\section{References}

[1] Shovlin, C. (2016) Up to Date. Hereditary Hemorrhagic Telangiectasia (Osler-Weber-Rendu Syndrome). In: Leung, L., Ed., Waltham.

[2] Sharathkumar, A.A. and Shapiro, A. (2008) Hereditary Haemorrhagic Telangiectasia. Haemophilia, 14, $1269-1280$. http://dx.doi.org/10.1111/j.1365-2516.2008.01774.x

[3] Guttmacher, A.E., Marchuk, D.A. and White, R.I. (1995) Hereditary Hemorrhagic Telangiectasia. New England Journal of Medicine, 333, 918-924. http://dx.doi.org/10.1056/NEJM199510053331407

[4] Assar, O.S., Friedman, C.M. and White, R.I. (1991) The Natural History of Epistaxis in Hereditary Hemorrhagic Telangiectasia. Laryngoscope, 101, 977-980. http://dx.doi.org/10.1288/00005537-199109000-00008

[5] Dines, D.E., et al. (1974) Pulmonary Arteriovenous Fistulas. Mayo Clinic Proceedings, 49, 460-465.

[6] McDonald, J. and Pyeritz, R. (2014) Hereditary Hemorrhagic Telangiectasia. University of Washington, Gene Reviews, Seattle.

[7] Bayrak-Toydemir, P., et al. (2006) A Fourth Locus for Hereditary Hemorrhagic Telangiectasia Maps to Chromosome 7. American Journal of Medical Genetics Part A, 140, 2155-2162. http://dx.doi.org/10.1002/ajmg.a.31450

[8] Kjeldsen, A.D., et al. (2005) Clinical Symptoms According to Genotype amongst Patients with Hereditary Haemorrhagic Telangiectasia. Journal of Internal Medicine, 258, 349-355. http://dx.doi.org/10.1111/j.1365-2796.2005.01555.x

[9] Gallione, C.J., et al. (2004) A Combined Syndrome of Juvenile Polyposis and Hereditary Haemorrhagic Telangiectasia Associated with Mutations in MADH4 (SMAD4). Lancet, 363, 852-859. http://dx.doi.org/10.1016/S0140-6736(04)15732-2

[10] Rigelsky, C.M., et al. (2008) BMPR2 Mutation in a Patient with Pulmonary Arterial Hypertension and Suspected Hereditary Hemorrhagic Telangiectasia. American Journal of Medical Genetics Part A, 146, 2551-2556. http://dx.doi.org/10.1002/ajmg.a.32468

[11] David, L., et al. (2007) Identification of BMP9 and BMP10 as Functional Activators of the Orphan Activin ReceptorLike Kinase 1 (ALK1) in Endothelial Cells. Blood, 109, 1953-1961. http://dx.doi.org/10.1182/blood-2006-07-034124

[12] Fernández-L, A., et al. (2006) Hereditary Hemorrhagic Telangiectasia, a Vascular Dysplasia Affecting the TGF-Beta Signaling Pathway. Clinical Medicine \& Research, 4, 66-78. http://dx.doi.org/10.3121/cmr.4.1.66

[13] González-Núñez, M., Muñoz-Félix, J.M. and López-Novoa, J.M. (2013) The ALK-1/Smad1 Pathway in Cardiovascular Physiopathology. A New Target for Therapy? Biochimica et Biophysica Acta, 1832, 1492-1510. http://dx.doi.org/10.1016/j.bbadis.2013.05.016

[14] HHT Mutation Database (2015) The University of Utah Department of Pathology and ARUP Laboratories. http://arup.utah.edu/database/HHT/

[15] Shoukier, M., et al. (2008) Characterization of Five Novel Large Deletions Causing Hereditary Haemorrhagic Telangiectasia. Clinical Genetics, 73, 320-330. http://dx.doi.org/10.1111/j.1399-0004.2008.00968.x

[16] Pousada, G., Baloira, A., Vilariño, C., Cifrian, M.J. and Valverde, D. (2014) Novel Mutations in BMPR2, ACVRL1 and KCNA5 Genes and Hemodynamic Parameters in Patients with Pulmonary Arterial Hypertension. PLoS ONE, 9, e100261. http://dx.doi.org/10.1371/journal.pone.0100261

[17] Gedge, F., et al. (2007) Clinical and Analytical Sensitivities in Hereditary Hemorrhagic Telangiectasia Testing and a 
Report of de Novo Mutations. The Journal of Molecular Diagnostics, 9, 258-265. http://dx.doi.org/10.2353/jmoldx.2007.060117

[18] Trembath, R.C., et al. (2001) Clinical and Molecular Genetic Features of Pulmonary Hypertension in Patients with Hereditary Hemorrhagic Telangiectasia. The New England Journal of Medicine, 345, 325-334. http://dx.doi.org/10.1056/NEJM200108023450503

[19] McDonald, J., et al. (2011) Molecular Diagnosis in Hereditary Hemorrhagic Telangiectasia: Findings in a Series Tested Simultaneously by Sequencing and Deletion/Duplication Analysis. Clinical Genetics, 79, 335-344. http://dx.doi.org/10.1111/j.1399-0004.2010.01596.x

[20] Olivieri, C., et al. (2007) Analysis of ENG and ACVRL1 Genes in 137 HHT Italian Families Identifies 76 Different Mutations (24 Novel). Comparison with Other European Studies. Journal of Human Genetics, 52, 820-829. http://dx.doi.org/10.1007/s10038-007-0187-5

[21] Lesca, G., et al. (2004) Molecular Screening of ALK1/ACVRL1 and ENG Genes in Hereditary Hemorrhagic Telangiectasia in France. Human Mutation, 23, 289-299. http://dx.doi.org/10.1002/humu.20017

[22] Lee, S.T., et al. (2009) Clinical Features and Mutations in the ENG, ACVRL1, and SMAD4 genes in Korean Patients with Hereditary Hemorrhagic Telangiectasia. Journal of Korean Medical Science, 24, 69-76. http://dx.doi.org/10.3346/jkms.2009.24.1.69

[23] Abdalla, S.A., Cymerman, U., Johnson, R.M., Deber, C.M. and Letarte, M. (2003) Disease-Associated Mutations in Conserved Residues of $A L K-1$ Kinase Domain. European Journal of Human Genetics, 11, 279-287. http://dx.doi.org/10.1038/sj.ejhg.5200919

[24] Bossler, A.D., Richards, J., George, C., Godmilow, L. and Ganguly, A. (2006) Novel Mutations in ENG and ACVRL1 Identified in a Series of 200 Individuals Undergoing Clinical Genetic Testing for Hereditary Hemorrhagic Telangiectasia (HHT): Correlation of Genotype with Phenotype. Human Mutation, 27, 667-675. http://dx.doi.org/10.1002/humu.20342

[25] Lesca, G., et al. (2006) Distribution of ENG and ACVRL1 (ALK1) Mutations in French HHT Patients. Human Mutation, 27, 598. http://dx.doi.org/10.1002/humu.9421

[26] Schulte, C., et al. (2005) High Frequency of ENG and ALK1/ACVRL1 Mutations in German HHT Patients. Human Mutation, 25, 595-602. http://dx.doi.org/10.1002/humu.9345

[27] Fernandez-L, A., et al. (2006) Mutation Study of Spanish Patients with Hereditary Hemorrhagic Telangiectasia and Expression Analysis of Endoglin and ALK1. Human Mutation, 27, 295. http://dx.doi.org/10.1002/humu.9413

[28] Lenato, G.M., et al. (2006) DHPLC-Based Mutation Analysis of ENG and ALK-1 Genes in HHT Italian Population. Human Mutation, 27, 213-214. http://dx.doi.org/10.1002/humu.9400

[29] Wehner, L.E., et al. (2006) Mutation Analysis in Hereditary Haemorrhagic Telangiectasia in Germany Reveals 11 Novel ENG and 12 Novel ACVRL1/ALK1 Mutations. Clinical Genetics, 69, 239-245. http://dx.doi.org/10.1111/j.1399-0004.2006.00574.x

[30] Tørring, P.M., Brusgaard, K., Ousager, L.B., Andersen, P.E. and Kjeldsen, A.D. (2014) National Mutation Study among Danish Patients with Hereditary Haemorrhagic Telangiectasia. Clinical Genetics, 86, 123-133. http://dx.doi.org/10.1111/cge.12269

[31] Bayrak-Toydemir, P., Mao, R., Lewin, S. and McDonald, J. (2004) Hereditary Hemorrhagic Telangiectasia: An Overview of Diagnosis and Management in the Molecular Era for Clinicians. Genetics in Medicine, 6, 175-191. http://dx.doi.org/10.1097/01.GIM.0000132689.25644.7C

[32] Brusgaard, K., et al. (2004) Mutations in Endoglin and in Activin Receptor-Like Kinase 1 among Danish Patients with Hereditary Haemorrhagic Telangiectasia. Clinical Genetics, 66, 556-561. http://dx.doi.org/10.1111/j.1399-0004.2004.00341.x

[33] Abdalla, S.A., et al. (2000) Analysis of $A L K-1$ and Endoglin in Newborns from Families with Hereditary Hemorrhagic Telangiectasia Type 2. Human Molecular Genetics, 9, 1227-1237. http://dx.doi.org/10.1093/hmg/9.8.1227

[34] Klaus, D.J., et al. (1998) Novel Missense and Frameshift Mutations in the Activin Receptor-Like Kinase-1 Gene in Hereditary Hemorrhagic Telangiectasia. Human Mutation, 12, 137. http://dx.doi.org/10.1002/(SICI)1098-1004(1998)12:2<137::AID-HUMU15>3.0.CO;2-M

[35] Olivieri, C., et al. (2002) Identification of 13 New Mutations in the ACVRL1 Gene in a Group of 52 Unselected Italian Patients Affected by Hereditary Haemorrhagic Telangiectasia. Journal of Medical Genetics, 39, E39. http://dx.doi.org/10.1136/jmg.39.7.e39

[36] Jia, J.J., et al. (2012) [Clinical Features and Screening of ACVRL1 Gene in II Hereditary Hemorrhagic Telangiectasia]. Chinese Medical Journal, 92, 1107-1111.

[37] Argyriou, L., et al. (2006) Novel Mutations in the ENG and ACVRL1 Genes Causing Hereditary Hemorrhagic Telean- 
giectasia. International Journal of Molecular Medicine, 17, 655-659. http://dx.doi.org/10.3892/ijmm.17.4.655

[38] Berg, J.N., et al. (1997) The Activin Receptor-Like Kinase 1 Gene: Genomic Structure and Mutations in Hereditary Hemorrhagic Telangiectasia Type 2. American Journal of Human Genetics, 61, 60-67. http://dx.doi.org/10.1086/513903

[39] Letteboer, T.G., et al. (2005) Hereditary Hemorrhagic Telangiectasia: ENG and ALK-1 Mutations in Dutch Patients. Human Genetics, 116, 8-16. http://dx.doi.org/10.1007/s00439-004-1196-5

[40] Olivieri, C., et al. (2006) Echocardiographic Screening Discloses Increased Values of Pulmonary Artery Systolic Pressure in 9 of 68 Unselected Patients Affected with Hereditary Hemorrhagic Telangiectasia. Genetics in Medicine, 8, 183-190. http://dx.doi.org/10.1097/01.gim.0000204463.77319.1c

[41] Kuehl, H.K., et al. (2005) Hepatic Manifestation Is Associated with ALK1 in Hereditary Hemorrhagic Telangiectasia: Identification of Five Novel ALK1 and One Novel ENG Mutations. Human Mutation, 25, 320. http://dx.doi.org/10.1002/humu.9311

[42] Kim, M.J., et al. (2011) Clinical and Genetic Analyses of three Korean Families with Hereditary Hemorrhagic Telangiectasia. BMC Medical Genetics, 12, 130. http://dx.doi.org/10.1186/1471-2350-12-130

[43] Komiyama, M., et al. (2014) Hereditary Hemorrhagic Telangiectasia in Japanese Patients. Journal of Human Genetics, 59, 37-41. http://dx.doi.org/10.1038/jhg.2013.113

[44] McDonald, J., et al. (2009) Multiple Sequence Variants in Hereditary Hemorrhagic Telangiectasia Cases: Illustration of Complexity in Molecular Diagnostic Interpretation. The Journal of Molecular Diagnostics, 11, 569-575. http://dx.doi.org/10.2353/jmoldx.2009.080148

[45] Richards-Yutz, J., Grant, K., Chao, E.C., Walther, S.E. and Ganguly, A. (2010) Update on Molecular Diagnosis of Hereditary Hemorrhagic Telangiectasia. Human Genetics, 128, 61-77. http://dx.doi.org/10.1007/s00439-010-0825-4

[46] Prigoda, N.L., et al. (2006) Hereditary Haemorrhagic Telangiectasia: Mutation Detection, Test Sensitivity and Novel Mutations. Journal of Medical Genetics, 43, 722-728. http://dx.doi.org/10.1136/jmg.2006.042606

[47] Fontalba, A., et al. (2008) Mutation Study of Spanish Patients with Hereditary Hemorrhagic Telangiectasia. BMC Medical Genetics, 9, 75. http://dx.doi.org/10.1186/1471-2350-9-75

[48] Abdalla, S.A., et al. (2005) Novel Mutations and Polymorphisms in Genes Causing Hereditary Hemorrhagic Telangiectasia. Human Mutation, 25, 320-321. http://dx.doi.org/10.1002/humu.9312

[49] Fernandez-L, A., et al. (2005) Blood Outgrowth Endothelial Cells from Hereditary Haemorrhagic Telangiectasia Patients Reveal Abnormalities Compatible with Vascular Lesions. Cardiovascular Research, 68, 235-248. http://dx.doi.org/10.1016/j.cardiores.2005.06.009

[50] Xie, G.L. and Li, Z.X. (2007) [Hereditary Hemorrhagic Telangiectasia Caused by Mutation in Intron 4 of ALK1 Gene: Analysis of a HTT Family]. National Medical Journal of China, 87, 249-252.

[51] Harrison, R.E., et al. (2003) Molecular and Functional Analysis Identifies $A L K-1$ as the Predominant Cause of Pulmonary Hypertension Related to Hereditary Haemorrhagic Telangiectasia. Journal of Medical Genetics, 40, 865-871. http://dx.doi.org/10.1136/jmg.40.12.865

[52] Chen, Y.J., et al. (2013) Clinical and Genetic Characteristics of Chinese Patients with Hereditary Haemorrhagic Telangiectasia-Associated Pulmonary Hypertension. European Journal of Clinical Investigation, 43, 1016-1024. http://dx.doi.org/10.1111/eci.12138

[53] Girerd, B., et al. (2010) Clinical Outcomes of Pulmonary Arterial Hypertension in Patients Carrying an ACVRL1 (ALK1) Mutation. American Journal of Respiratory and Critical Care Medicine, 181, 851-861. http://dx.doi.org/10.1164/rccm.200908-12840C

[54] Canzonieri, C., et al. (2014) Endoscopic Evaluation of Gastrointestinal Tract in Patients with Hereditary Hemorrhagic Telangiectasia and Correlation with Their Genotypes. Genetics in Medicine, 16, 3-10. http://dx.doi.org/10.1038/gim.2013.62

[55] Giordano, P., et al. (2006) Screening for Children from Families with Rendu-Osler-Weber Disease: From Geneticist to Clinician. Journal of Thrombosis and Haemostasis, 4, 1237-1245. http://dx.doi.org/10.1111/j.1538-7836.2006.01934.x

[56] Johnson, D.W., et al. (1995) A Second Locus for Hereditary Hemorrhagic Telangiectasia Maps to Chromosome 12. Genome Research, 5, 21-28. http://dx.doi.org/10.1101/gr.5.1.21

[57] Brakensiek, K., et al. (2008) Detection of a Significant Association between Mutations in the ACVRL1 Gene and Hepatic Involvement in German Patients with Hereditary Haemorrhagic Telangiectasia. Clinical Genetics, 74, 171-177. http://dx.doi.org/10.1111/j.1399-0004.2008.01029.x

[58] Chida, A., et al. (2012) Outcomes of Childhood Pulmonary Arterial Hypertension in BMPR2 and ALK1 Mutation Carriers. American Journal of Cardiology, 110, 586-593. http://dx.doi.org/10.1016/j.amjcard.2012.04.035

[59] Assis, A.M., Costa. F.F., Arruda, V.R., Annichino-Bizzacchi, J.M. and Bertuzzo, C.S. (2007) Three Novel Mutations 
in the Activin Receptor-Like Kinase $1(A L K-1)$ Gene in Hereditary Hemorrhagic Telangiectasia Type 2 in Brazilian Patients. Journal of Human Genetics, 52, 237-243. http://dx.doi.org/10.1007/s10038-006-0104-3

[60] Fujiwara, M., et al. (2008) Implications of Mutations of Activin Receptor-Like Kinase 1 Gene (ALK1) in Addition to Bone Morphogenetic Protein Receptor II Gene (BMPR2) in Children with Pulmonary Arterial Hypertension. Circulation Journal, 72, 127-133. http://dx.doi.org/10.1253/circj.72.127

[61] Bayrak-Toydemir, P., et al. (2006) Genotype-Phenotype Correlation in Hereditary Hemorrhagic Telangiectasia: Mutations and Manifestations. American Journal of Medical Genetics Part A, 140A, 463-470. http://dx.doi.org/10.1002/ajmg.a.31101

[62] Sankelo, M., Halme, M., Laitinen, T. and Mattila, P.S. (2008) Hereditary Hemorrhagic Telangiectasia Type 1 and 2 Mutations in Finland. Acta Oto-Laryngologica, 128, 1238-1241. http://dx.doi.org/10.1080/00016480801908035

[63] Abdalla, S.A., et al. (2004) Primary Pulmonary Hypertension in Families with Hereditary Haemorrhagic Telangiectasia. European Respiratory Society, 23, 373-377. http://dx.doi.org/10.1183/09031936.04.00085504

[64] Eyries, M., et al. (2012) ACVRL1 Germinal Mosaic with Two Mutant Alleles in Hereditary Hemorrhagic Telangiectasia Associated with Pulmonary Arterial Hypertension. Clinical Genetics, 82, 173-179. http://dx.doi.org/10.1111/j.1399-0004.2011.01727.x

[65] Jones, G., Robertson, L., Harrison, R., Ridout, C. and Vasudevan, P. (2014) Somatic Mosaicism in ACVRL1 with Transmission to Several Offspring Affected with Severe Pulmonary Arterial Hypertension. American Journal of Medical Genetics Part A, 164, 2121-2123. http://dx.doi.org/10.1002/ajmg.a.36568

\section{Submit or recommend next manuscript to SCIRP and we will provide best service for you:}

Accepting pre-submission inquiries through Email, Facebook, Linkedin, Twitter, etc A wide selection of journals (inclusive of 9 subjects, more than 200 journals)

Providing a 24-hour high-quality service

User-friendly online submission system

Fair and swift peer-review system

Efficient typesetting and proofreading procedure

Display of the result of downloads and visits, as well as the number of cited articles

Maximum dissemination of your research work

Submit your manuscript at: http://papersubmission.scirp.org/ 